\title{
BOREL DIRECTION RELATIVE TO FUNCTION-VALUES OF MEROMORPHIC FUNCTIONS WITH FINITE LOGARITHMIC ORDER
}

\author{
PETER TIEN-YU CHERN
}

\begin{abstract}
It is shown that if $f(z)$ is meromorphic in the complex plane $\mathbb{C}$ with finite positive logarithmic order $\lambda$ and its characteristic function $T(r, f)$ satisfies the growth condition

$$
\limsup _{r \rightarrow+\infty} T(r, f) /(\log r)^{2}=+\infty,
$$

then there is a number $\theta$ with $0 \leq \theta<2 \pi$ such that for each positive number $\epsilon$, the expression

$$
\limsup _{r \rightarrow+\infty} \frac{\log \left\{\sum_{i=1}^{3} n\left(r, \theta, \epsilon, f=a_{i}(z)\right)\right\}}{\log \log r}=\lambda-1,
$$

holds for any three distinct meromorphic functions $a_{i}(z)(i=1,2,3)$ with $T\left(r, a_{i}\right)=o(U(r, f) /$ $\left.(\log r)^{2}\right)$, as $r \rightarrow+\infty$, where $n(r, \varphi, \epsilon, f=a(z))$ denotes the number of roots counting multiplicities of the equation $f(z)=a(z)$ for $z$ in the angular domain $\Omega(r, \varphi, \epsilon)=\{z:|\arg z-\varphi|<\epsilon$, $|z|<r\}$ where $0 \leq \varphi<2 \pi, \epsilon>0, U(r, f)=(\log r)^{\lambda(r)}$, and $\limsup \lambda(r)=\lambda$.
\end{abstract}

\section{Introduction and Results}

In 1] we systematically establish basic results on the value distribution for a class of transcendental meromorphic functions of finite logarithmic order. Here we shall deal with the argument distribution for them.

Let $f(z)$ be meromorphic in the complex plane $\mathbb{C}$. We denote by $T(r, f)$ the Nevanlinna characteristic function of $f(z)$. If $f(z)$ is of zero order, the number

$$
\limsup _{r \rightarrow+\infty} \frac{\log T(r, f)}{\log \log r}
$$

is called the logarithmic order [1] of $f(z)$. Throughout this paper, let $n(r, \varphi, \epsilon, f=a(z))$ denote the number of roots counting multiplicities of the equation $f(z)=a(z)$ for $z$ in

Received September 26, 2005.

2000 Mathematics Subject Classification. Primary 30D30, 30D35.

Key words and phrases. Borel direction, function-values, finite logarithmic order.

This research was supported in part by the NSC R.O.C. under the grant nsc 85-2115-M-214001, a fund from Academia Sinica (Taipei, Taiwan) and a fund from Michigan State University, U.S.A. 
the angular domain $\Omega(r, \varphi, \epsilon)=\{z:|\arg z-\varphi|<\epsilon,|z|<r\}$ where $0 \leq \varphi<2 \pi, \epsilon>0$, and $N(r, \varphi, \epsilon, f=a(z))$ denote the integral counting function of $f(z)$ associated with $n(r, \varphi, \epsilon, f=a(z))$.

In 1928, G. Valiron [6] first proved the existence of a Borel direction for any meromorphic function of finite positive order. Later for meromorphic functions of zero order, he obtained the following results:

Theorem A.([7, p.300]) If $f(z)$ is a meromorphic function with zero order in $\mathbb{C}$ satisfying

$$
\limsup _{r \rightarrow+\infty} \frac{T(r, f)}{(\log r)^{2} \log \log r}=+\infty
$$

then there exists a continuous function $U(r)$ such that
(a) $\limsup _{r \rightarrow+\infty} \frac{T(r, f)}{U(r)}=1$,
(b) $\limsup _{r \rightarrow+\infty} \frac{U(r)}{(\log r)^{2} \log \log r}=+\infty$,
(c) $\frac{U(2 r)}{U(r)} \leq K<+\infty, \quad r \geqq r_{o}$.

Theorem B. ([7, p.306]) If $f(z)$ is given in Theorem $A$, then there exists a $\theta \in[0,2 \pi)$ for each $a \in \widehat{\mathbb{C}}=\mathbb{C} \cup\{\infty\}$, the inequality

$$
\delta(\theta, f=a)=: \limsup _{\epsilon \rightarrow 0} \limsup _{r \rightarrow+\infty} \frac{\pi N(r, \theta, \epsilon, f=a)}{\epsilon U(r)}>0
$$

holds with at most two possible exceptional values in $\widehat{\mathbb{C}}$.

$\delta(\theta, f=a)$ (in Theorem B) is called the characteristic of the value a with respect to the direction $\Delta(\theta)=\left\{r e^{i \theta} \mid r \geq 0\right\}$ of $f(z)$.

Theorem C.([8, p.38, Theorem 35]) If $f(z)$ is given as in Theorem A, then there exists $a \theta \in[0,2 \pi)$ for each $\epsilon>0$, for each $a \in \mathbb{C}$, the inequality

$$
\limsup _{r \rightarrow+\infty} \frac{n(r, \theta, \epsilon, f=a)}{r U^{\prime}(r)}>0
$$

holds with at most two possible exceptional values in $\widehat{\mathbb{C}}$.

Recently the author [2] has proved the existence of a Borel direction for meromorphic function of finite logarithmic order and obtained the following result:

Theorem D. ([2, Theorem 2]) Let $f(z)$ be a meromorphic function in the complex plane $\mathbb{C}$ with finite logarithmic order $\lambda$. If $f(z)$ satisfies the growth condition

$$
\limsup _{r \rightarrow+\infty} T(r, f) /(\log r)^{2}=+\infty,
$$


then there exists a ray $\Delta(\theta)=\{z: \arg z=\theta\}, 0 \leq \theta<2 \pi$, with $0 \leq \theta<2 \pi$ such that for every small positive number $\epsilon\left(<\frac{\pi}{2}\right)$ and every $a \in \widehat{\mathbb{C}}=\mathbb{C} \cup\{\infty\}$, the expression

$$
\limsup _{r \rightarrow+\infty} \frac{\log n(r, \theta, \epsilon, f=a)}{\log \log r}=\lambda-1,
$$

holds with at most two possible exceptional values of $a$.

In this article we establish the existence of a Borel direction relative to function-values for any meromorphic function of finite logarithmic order with the growth condition (1.5) which may extend and sharpen Valiron's results above and the author's result Theorem D above.

To this end we need an important tool, viz., a proximate logarithmic order.

Definition. If $f(z)$ is a meromorphic function in $\mathbb{C}$ with finite logarithmic order $\lambda$. A nonnegative continuous function $\lambda(r)$ defined in $(0,+\infty)$ is called a proximate logarithmic order of $T(r, f)$, if $\lambda(r)$ satisfies the following properties:

(1) $\limsup _{r \rightarrow+\infty} \lambda(r)=\lambda$,

(2) $\lambda^{\prime}(r)$ exists everywhere in $(0,+\infty)$ except possibly in a countable set where $\lambda^{\prime}\left(r^{+}\right)$ and $\lambda^{\prime}\left(r^{-}\right)$exist. Furthermore, if we use one-sided derivatives $\lambda^{\prime}\left(r^{+}\right)$or $\lambda^{\prime}\left(r^{-}\right)$ instead of $\lambda^{\prime}(r)$ for $r$ in the exceptional set, then we have

$$
\lim _{r \rightarrow+\infty} r(\log r) \lambda^{\prime}(r) \log \log r=0 .
$$

(3) Let $U(r, f)=(\log r)^{\lambda(r)}$, we have $T(r, f) \leq U(r, f)$ for sufficiently large $r$, and $\lim \sup _{r \rightarrow+\infty} T(r, f) / U(r, f)=1$.

The above $U(r, f)$ defined in (3) is called a logarithmic type function of $T(r, f)$ and is more general and sharper than $U(r)$ which was constructed by Valiron for $f(z)$ satisfying the growth condition (1.2). (This assumes such a $\lambda(r)$ exists.)

The author obtains the following result.

Theorem E.([1, Theorem 2.1]) If $f(z)$ is a transcendental meromorphic function in $\mathbb{C}$ with finite positive logarithmic order $\lambda$, then $T(r, f)$ has a proximate logarithmic order $\lambda(r)$.

For a meromorphic function of finite logarithmic order, Theorem $\mathrm{E}$ is a sharper result of above Theorem A.

We now state the main results of this article as follows:

Theorem 1. If $f(z)$ is a meromorphic function in $\mathbb{C}$ with finite positive logarithmic order $\lambda$ and satisfies the growth condition (1.5), then there exists a number $\theta$ with $0 \leq$ 
$\theta<2 \pi$ such that for each positive number $\epsilon$, the equality

$$
\limsup _{r \rightarrow+\infty} \frac{\log \left\{\sum_{i=1}^{3} N\left(r, \theta, \epsilon, f=a_{i}(z)\right)\right\}}{\log \log r}=\lambda,
$$

holds for any three distinct meromorphic functions $a_{i}(z)(i=1,2,3)$ with $T\left(r, a_{i}\right)=$ $o\left(U(r, f) /(\log r)^{2}\right)$, as $r \rightarrow+\infty$, where $U(r, f)$ is a logarithmic type function of $T(r, f)$.

Theorem 2. If $f(z)$ and $U(r, f)$ are given as in Theorem 1, then there is a number $\theta$ with $0 \leq \theta<2 \pi$ such that for each positive number $\epsilon$, the inequality

$$
\limsup _{r \rightarrow+\infty} \sum_{i=1}^{3} N\left(r, \theta, \epsilon, f=a_{i}(z)\right) / U(r, f)>0,
$$

holds for any three distinct meromorphic functions $a_{i}(z)(i=1,2,3)$ with $T\left(r, a_{i}\right)=$ $o\left(U(r, f) /(\log r)^{2}\right)$, as $r \rightarrow+\infty$.

For meromorphic functions of finite logarithmic order, this Theorem 2 extends Valiron's Theorem B above.

Theorem 3. (The existence theorem of a Borel direction relative to function-values for meromorphic functions with finite logarithmic order) If $f(z)$ and $U(r, f)$ are given as in Theorem 1 , then there is a number $\theta$ with $0 \leq \theta<2 \pi$ such that for each positive number $\epsilon$, the inequality

$$
\limsup _{r \rightarrow+\infty} \sum_{i=1}^{3} n\left(r, \theta, \epsilon, f=a_{i}(z)\right) \log r / U(r, f)>0
$$

and the equation

$$
\limsup _{r \rightarrow+\infty} \frac{\log \left\{\sum_{i=1}^{3} n\left(r, \theta, \epsilon, f=a_{i}(z)\right)\right\}}{\log \log r}=\lambda-1 .
$$

both hold for any three distinct meromorphic functions $a_{i}(z)(i=1,2,3)$ with $T\left(r, a_{i}\right)=$ $o\left(U(r, f) /(\log r)^{2}\right)$, as $r \rightarrow+\infty$.

For functions of finite logarithmic order, the expression (1.9) of Theorem 3 extends the above Valiron's Theorem C since the inequality (1.9) implies the inequality (1.4) and the expression (1.10) of the above Theorem 3 extends the above author's Theorem D.

Theorem 4. If $f(z)$ and $U(r, f)$ are given as in Theorem 1 , then for each positive number $\epsilon$, there exists a number $\theta$ with $0 \leq \theta<2 \pi$ such that the inequality (1.8) holds for any three distinct meromorphic functions $a_{i}(z)(i=1,2,3)$ with $T\left(r, a_{i}\right)=$ $o\left(U(r, f) /(\log r)^{2}\right)$, as $r \rightarrow+\infty$. 


\section{Remarks.}

1. A ray $\Delta(\theta)=\{z: \arg z=\theta\}$ satisfying (1.7) in Theorem 1 if and only if it satisfies (1.10) in Theorem 3, since $n(r, f=a)$ has logarithmic order $\lambda-1$ if and only if $N(r, f=a)$ has logarithmic order $\lambda$ (see [1, Theorem 4.1]).

2. A. Ostrowski [4] constructed a transcendental meromorphic function $f(z)$ such that $T(r, f)=O\left((\log r)^{2}\right)$, but this $f(z)$ has no Julia direction. These reflect that the growth condition (1.5) cannot be weakened in Theorem 1, Theorem 2 and Theorem 3.

3. If $f(z)$ and $a_{i}(z)(i=1,2,3)$ are given as in Theorem 1 , by a result in Chern 1 , Theorem 4.1], the logarithmic order of $n(r, f=a) \leq \lambda-1$, for each $a \in \widehat{\mathbb{C}}$, hence we have

$$
\limsup _{r \rightarrow+\infty} \frac{\log \left\{\sum_{i=1}^{3} n\left(r, \theta, \epsilon, f=a_{i}(z)\right)\right\}}{\log \log r} \leq \lambda-1 .
$$

To prove (1.10), it suffices to show the inequality

$$
\limsup _{r \rightarrow+\infty} \frac{\log \left\{\sum_{i=1}^{3} n\left(r, \theta, \epsilon, f=a_{i}(z)\right)\right\}}{\log \log r} \geq \lambda-1 .
$$

holds for any three distinct meromorphic functions $a_{i}(z)(i=1,2,3)$ with $T\left(r, a_{i}\right)=$ $o\left(U(r, f) /(\log r)^{2}\right)$, as $r \rightarrow+\infty$. The inequality (1.13) is a consequence of the inequality (1.10) in Theorem 3.

4. To prove Theorem 3, it suffices to prove (1.8) in Theorem 2, since (1.9) implies (1.12), (1.11) and (1.12) imply (1.10) and for any $a \in \widehat{\mathbb{C}}$

$$
\frac{1}{\lambda} \limsup _{r \rightarrow+\infty} \frac{n(r, \theta, \epsilon, f=a) \log r}{U(r, f)} \geq \limsup _{r \rightarrow+\infty} \frac{N(r, \theta, \epsilon, f=a)}{U(r, f)},
$$

(see [3, Lemma 1]). This is essentially an application of L'Hôpital's rule.

5. Theorem 4 implies Theorem 2. The proof is as follows.

Let $\epsilon_{m}(m=1,2, \ldots)$ be a sequence of numbers satisfying $0<\epsilon_{m}<\frac{\pi}{2}$ and $\lim _{m \rightarrow+\infty} \epsilon_{m}=0$. By Theorem 4, for each $\epsilon_{m}$ there is a number $\theta_{m} \in[0,2 \pi)$ for each $a(z) \in \mathcal{A}=\left\{a(z): a(z)\right.$ is meromorphic in $\mathbb{C}$ with $\left.T(r, a(z))=o\left(U(r, f) /(\log r)^{2}\right)\right\}$, we have

$$
\limsup _{r \rightarrow+\infty} \frac{N\left(r, \theta_{m}, \epsilon_{m}, f=a(z)\right)}{U(r, f)}>0,
$$

with at most two exceptional function $a(z) \in \mathcal{A}$.

Let $\theta_{0}$ be a limit point of $\left\{\theta_{m}\right\}_{m=1}^{+\infty}$, for any $\epsilon, 0<\epsilon<\frac{\pi}{2}$, there is a sufficiently large integer $m$ such that

$$
\left|\theta_{m}-\theta_{0}\right|<\frac{\epsilon}{2} \quad \text { and } \quad \epsilon_{m}<\frac{\epsilon}{2} .
$$


This yields

$$
\limsup _{r \rightarrow+\infty} \frac{N\left(r, \theta_{0}, \epsilon, f=a(z)\right)}{U(r, f)} \geq \limsup _{r \rightarrow+\infty} \frac{N\left(r, \theta_{m}, \epsilon_{m}, f=a(z)\right)}{U(r, f)}>0
$$

with at most two possible exceptional functions $a(z) \in \mathcal{A}$.

6. There needs to be a gap between the growth $T(r, f)$ and $T(r, a(z))$ where $a(z) \in \mathcal{A}$. This comes from the error term of the angular second fundamental Theorem of Tsuji [5, Lemma, p.277].

The remainder is to prove Theorem 4.

\section{The Proof of Theorem 4}

Let $S(r, \varphi, \alpha, f)$ be the spherical area of the image under $f(z)$ of $\Omega(r, \varphi, \alpha), T_{o}(r, \varphi, \alpha, f)$ be the Ahlfors-Shimizu characteristic of $f(z)$ associated with $S(r, \varphi, \alpha, f)$. If $\alpha \geq \pi$, we put $T_{o}(r, \varphi, \alpha, f)=T_{o}(r, f)$. Suppose that the conclusion of Theorem 4 is incorrect, then there exists a positive number $\alpha$, for each $\varphi, 0 \leq \varphi<2 \pi$, there exist three distinct meromorphic functions $a_{\varphi_{j}}(j=1,2,3)$ with $T\left(r, a_{\varphi_{j}}\right)=o\left(U(r, f) /(\log r)^{2}\right)$ such that the expression

$$
\limsup _{r \rightarrow+\infty} \sum_{j=1}^{3} N\left(r, \varphi, \alpha, f=a_{\varphi_{j}}(z)\right) / U(r, f)=0
$$

holds.

Since the family of open intervals $\chi=\{(\varphi-\alpha / 4, \varphi+\alpha / 4): \varphi \in[0,2 \pi]\}$ is an open covering of the closed interval $[0,2 \pi]$, there exists a finite subcovering $\chi_{o}=\left\{\left(\varphi_{k}-\alpha / 4\right.\right.$, $\left.\left.\varphi_{k}+\alpha / 4\right): k=1, \ldots, n\right\}$ which covers $[0,2 \pi]$.

For each positive integer $k,(1 \leq k \leq n)$, we put

$$
F_{k}(z)=\left(f(z)-a_{\varphi_{k 1}}(z)\right)\left(a_{\varphi_{k 3}}(z)-a_{\varphi_{k 2}}(z)\right) /\left(f(z)-a_{\varphi_{k 2}}(z)\right)\left(a_{\varphi_{k 3}}(z)-a_{\varphi_{k 1}}(z)\right),
$$

where $a_{\varphi_{k j}}(z),(j=1,2,3)$ depending on $\varphi_{k}$ and $\alpha$ and satisfying the expression (2.1). The function $f$ can be written as

$$
f=\frac{g_{\varphi_{k 1}} F_{k}+g_{\varphi_{k 2}}}{g_{\varphi_{k 3}} F_{k}+g_{\varphi_{k 4}}}
$$

For the above expression (2.3), applying a result of Tsuji [5,Lemma, p.277], we have

$$
S\left(r, \varphi_{k}, \alpha / 4, f\right) \leq 27 S\left(64 r, \varphi_{k}, \alpha / 2, F_{k}\right)+O\left(\int_{1}^{128 r}\left(\sum_{j=1}^{4} T_{o}\left(t, g_{\varphi_{k j}}\right) / t\right) d t\right)
$$

Since $\left(\sum_{j=1}^{4} T_{o}\left(t, g_{\varphi_{k j}}\right)=o\left(U(r, f) /(\log r)^{2}\right)\right.$, it follows that

$$
S\left(r, \varphi_{k}, \alpha / 4, f\right) \leq 27 S\left(64 r, \varphi_{k}, \alpha / 2, F_{k}\right)+o(U(r, f) / \log r)
$$


Dividing two sides of the above inequality (2.5) by $r$, then integrating them to $r$, we have

$$
T_{o}\left(r, \varphi_{k}, \alpha / 4, f\right) \leq 27 T_{o}\left(64 r, \varphi_{k}, \alpha / 2, F_{k}\right)+o(U(r, f)) .
$$

Since $f$ satisfying the growth condition (1.5), by a result of Tsuji Chern 1, Theorem VII.3, p.272], we have

$$
T_{o}\left(r, \varphi_{k}, \alpha / 4, f\right) \leq 81 \sum_{j=1}^{3} N\left(128 r, \varphi_{k}, \alpha, F_{k}=b_{j}\right)+o(U(r, f))
$$

where $b_{1}=0, b_{2}=\infty, b_{3}=1$.

Since $\chi_{o}$ covers $[0,2 \pi]$, we have

$$
\begin{aligned}
T_{o}(r, f) & \leq \sum_{k=1}^{n} T_{o}\left(r, \varphi_{k}, \alpha / 4, f\right) \\
& \leq 81 \sum_{k=1}^{n} \sum_{j=1}^{3} N\left(128 r, \varphi_{k}, \alpha, F_{k}=b_{j}\right)+o(U(r, f)) \\
& =81 \sum_{k=1}^{n} \sum_{j=1}^{3} N\left(128 r, \varphi_{k}, \alpha, f=a_{\varphi_{k j}}\right)+o(U(r, f)) .
\end{aligned}
$$

Dividing two sides of the above inequality $(2.8)$ by $U(r, f)$, then taking $\limsup _{r \rightarrow+\infty}$ we have

$$
\begin{aligned}
\limsup _{r \rightarrow+\infty} T_{o}(r, f) / U(r, f) & \leq 81 \sum_{k=1}^{n} \limsup _{r \rightarrow+\infty} \sum_{j=1}^{3} \frac{N\left(128 r, \varphi_{k}, \alpha, f=a_{\varphi_{k j}}\right)}{U(r, f)} \\
& =81 \sum_{k=1}^{n} \limsup _{r \rightarrow+\infty} \sum_{j=1}^{3} \frac{N\left(128 r, \varphi_{k}, \alpha, f=a_{\varphi_{k j}}\right)}{U(128 r, f)}=0 .
\end{aligned}
$$

The above result $(2.9)$ contradicts to $\limsup _{r \rightarrow+\infty} T(r, f) / U(r, f)=1$, and $T_{o}(r, f) \sim T(r, f)$. This completes the proof of Theorem 4.

\section{References}

[1] Peter T. Y. Chern, On meromorphic functions of finite logarithmic order, Trans. Amer. Math. Soc. 358(2006), 473-489.

[2] Peter T. Y. Chern, Common Borel Directions of a meromorphic function with zero order and its derivative, Proc. Amer. Math. Soc. 132(2004), 1171-1175.

[3] Peter T. Y. Chern, On Erdös problem about a deficiency relation, Bull. Inst. Math. Academia Sinica 28(2000), 87-92.

[4] A. Ostrowski, Über Folgen analytisher Funktionen und einige Verscharfungen des Picardschen Satzes, Math. Zeit. 24(1926), 215-258. 
[5] M. Tsuji, Potential Theory in Modern Function Theory, Chelsea Publ. N. Y. 1975.

[6] G. Valiron, Recherches sur le theoreme de Borel dans la theorie des fonctions meromorphes, Acta Math. 52 (1928), 67-92.

[7] G. Valiron, Sur les directions de Borel des functions meromorphes d'ordre nul, Bull. Sc. Math., (1935), 298-320.

[8] G. Valiron, Direction de Borel des fonctions meromorphes, Memorial Sc. Math. Fasc. 89, Paris, Gauthier-Villars, 1938.

Department of Applied Mathematics, I-Shou University, Ta Hsu, Kaohsiung 84099, Taiwan.

E-mail: tychern@mail.isu.edu.tw 